\title{
Nanoscale Calorimetry of Isolated Polyethylene Single Crystals
}

\author{
A. T. KWAN, M. YU. EFREMOV, E. A. OlSON, F. SCHIETTEKATTE, M. ZHANG, P. H. GEIL, L. H. ALLEN \\ Materials Science and Engineering and Coordinated Science Laboratory, University of Illinois at Urbana-Champaign, \\ Urbana, Illinois 61801
}

Received 1 December 2000; accepted 22 March 2001

\begin{abstract}
We used thin-film differential scanning calorimetry to investigate the melting of isolated polyethylene single crystals with lamellar thicknesses of $12 \pm 1 \mathrm{~nm}$. We observed the melting of as few as 25 crystals. Over a wide number of crystals (25-2000 crystals), the heat of fusion was $40 \%$ larger than the bulk value. The melting temperature of the isolated single crystals was $123 \pm 2{ }^{\circ} \mathrm{C}, 9{ }^{\circ} \mathrm{C}$ lower than that of the bulk material. We also measured the heat of fusion of quenched crystals $( \pm 15 \%)$ over a wide range of heating rates $(20,000-100,000 \mathrm{~K} / \mathrm{s})$. Annealing the quenched crystals resulted in shifts in the endotherm peak by as much as $15^{\circ} \mathrm{C}$. (C) $2001 \mathrm{John}$ Wiley \& Sons, Inc. J Polym Sci B: Polym Phys 39: 1237-1245, 2001
\end{abstract}

Keywords: microelectromechanical systems; calorimetry; nanocalorimetry; polyethylene single crystals; melting

\section{INTRODUCTION}

We introduce a new high-sensitivity scanning calorimetry technique with ultrafast scan rates for investigations of natural and synthetic macromolecular systems at the nanometer scale. We use a recently developed nanocalorimeter technique that has sensitivity on the nanojoule level with scan rates of over $100,000 \mathrm{~K} / \mathrm{s}$ and has the capability of studying samples as small as $10 \mathrm{pg}{ }^{1}$ Furthermore, the sample holder is thin enough to allow direct transmission electron microscopy (TEM) measurements. In this article, we demonstrate the capabilities of the device with a study on the melting and annealing characteristics of lamellar single crystals of polyethylene (PE). We obtain thickness, diffraction, and calorimetry data on the same set of isolated PE crystals.

$\mathrm{PE}$ is the model crystalline system in polymer science, and yet there still remain important is-

Correspondence to: L. H. Allen (E-mail: l-allen9@uiuc.edu) Journal of Polymer Science: Part B: Polymer Physics, Vol. 39, 1237-1245 (2001) ○ 2001 John Wiley \& Sons, Inc. sues that cannot be addressed via conventional calorimetry. These include variations of the melting point $\left(T_{\mathrm{m}}\right)$ as a function of both initial thickness ${ }^{2}$ and thermal cycling history. ${ }^{3} \mathrm{PE}$ single crystals are metastable: they reorganize and recrystallize during the process of the calorimetry scan itself. ${ }^{4-6}$ The crystals also tend to thicken during heating. ${ }^{7}$

An ideal sample configuration would have PE single crystals, grown from solution, sprayed onto a thin substrate. After the solvent vaporizes, the residual PE crystals lie flat on the substrate and are isolated from one another. Isolation limits any interactions between the crystals, allows convenient thickness measurements with atomic force microscopy (AFM), and permits diffraction on individual crystals to be taken with TEM.

Such a sample configuration is not possible with conventional differential scanning calorimetry (DSC), which is intended for macroscale samples. Therefore, conventional DSC does not have the sensitivity needed to detect a small enough number of PE single crystals so that they are isolated. Thus, macroscale DSC has been limited 
to sample sizes no smaller than a fraction of a milligram. ${ }^{8,9}$

Because of the crystals' tendency to recrystallize after melting on slow heating, the $T_{\mathrm{m}}$ of PE single crystals cannot be easily obtained directly. ${ }^{10}$ At the typical heating rates $(10-20 \mathrm{~K} / \mathrm{min})$ employed in macroscale DSC, there is no simple correlation between the measured $T_{\mathrm{m}}$ and the properties of the original material. A possible solution to this problem would be to use fast scan rates. ${ }^{4}$ However, at faster scan rates an intrinsic thermal lag persists with DSC that causes a shift of the melting endotherm to higher temperatures. ${ }^{2}$

Our nanocalorimeter has several advantages over conventional DSC in regard to studying $\mathrm{PE}$ single crystals. First, by reducing the overall thermal mass of the calorimetric system (much smaller than DSC), it becomes very sensitive. Our device is sensitive to the nanojoule level, ${ }^{11}$ which corresponds to approximately hundreds of picograms of PE single crystals ( $\sim 25$ individual crystals). Second, because it employs very fast scan rates (up to $1,000,000 \mathrm{~K} / \mathrm{s}$ ) during calorimetric measurements, recrystallization before melting is inhibited. The scan rates we employ, $10^{4}$ to $10^{5}$ $\mathrm{K} / \mathrm{s}$, are 3 orders of magnitude greater than those used in optical microscopy experiments, which are able to detect melting at fast heating rates $(2-33 \mathrm{~K} / \mathrm{s}) .{ }^{12}$ In addition, because of the intimate thermal contact between the sample and heater thermometer in our design, the problem of thermal lag is drastically reduced. Finally, the nanocalorimeter is constructed with a thin silicon nitride membrane as a sample holder. This membrane is an ideal substrate for isolated PE single crystals to be observed with AFM and TEM.

Previously, this technique and others similar to it have been used to study nanometer-sized metal particles, ${ }^{11,13}$ thin films, ${ }^{14}$ nanogram amounts of $n$-alkanes, ${ }^{15}$ nanoliter-sized liquid samples ${ }^{16}$ and individual superconductor crystals. ${ }^{17,18}$ No work has been done to our knowledge on the investigation of polymer single crystals with nanocalorimetry.

The effect of annealing on the thermodynamics of the PE system would also be of considerable interest. Past work has been done with X-ray techniques, ${ }^{7,19}$ Raman spectroscopy, ${ }^{3,20}$ and neutron scattering ${ }^{21}$ in the analysis of annealed single crystals. It would be quite interesting to study annealed crystals with our technique. In this article, we only present the calorimetry of annealed, quenched crystals.

\section{TDSC sensor (not to scale)}

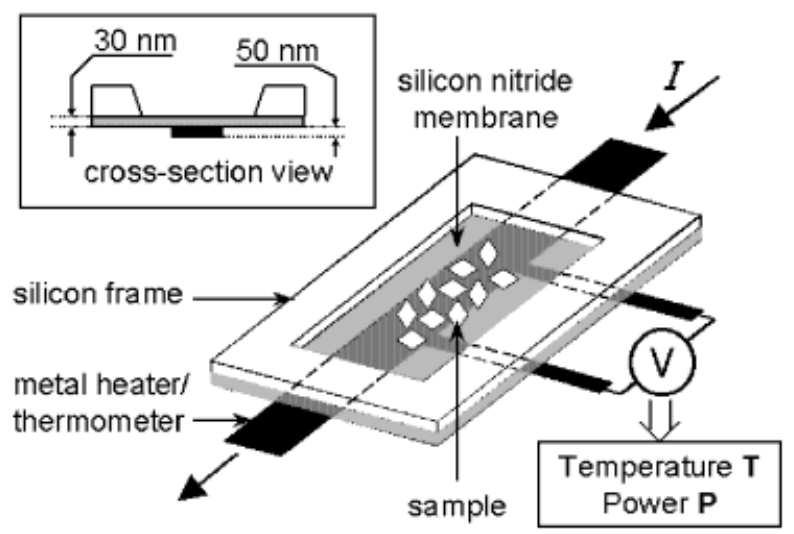

Figure 1. Schematic of the thin-film differential scanning calorimeter. A sample of PE single crystals is deposited on top of the heater area. Included is a crosssection view of the sensor. Relative dimensions have been exaggerated for clarity.

\section{EXPERIMENTAL}

\section{Nanocalorimetry}

The thin-film differential scanning calorimetry (TDSC) method relies on microelectromechanical calorimetric sensors. The calorimeters described in this work were fabricated similarly to those we previously reported. ${ }^{1}$ The planar and cross-sectional configurations of the TDSC system are shown in Figure 1. The sensors consisted of an extremely thin $(30 \mathrm{~nm})$ amorphous silicon nitride membrane $\left(\mathrm{a}-\mathrm{Si}_{3} \mathrm{~N}_{4-x}\right)$ supported by a silicon frame.

On one side of the membrane, a patterned thin $(50 \mathrm{~nm})$ platinum strip (500 $\mu \mathrm{m} \times 5 \mathrm{~mm}, \sim 70 \Omega$ ) was deposited and used simultaneously as a heater and resistive thermometer during the experiments. The material of interest was deposited on the silicon nitride side so that it rested above the heater/thermometer. Differential calorimetry was achieved with two identical sensors in one setup: a sample sensor (with material) and a reference sensor (with no material). Before the experiment, both sensors were calibrated in a furnace. As a control experiment, indium was deposited on the sensor, at a thickness where sizedependent melting did not occur, ${ }^{22}$ and its $T_{\mathrm{m}}$ was recorded as another means of calibrating the sensor for accurate temperature measurement.

The calorimetric measurement was initiated with the application of a synchronized direct-current electrical pulse (9-25 $\mathrm{mA}$ and $2-10 \mathrm{~ms}$ long) 
to each metal heater. The temperature of the sensors increased by joule heating and typically reached $200{ }^{\circ} \mathrm{C}$ at the end of the pulse. High heating rates $\left(2 \times 10^{4}\right.$ to $\left.10^{5}{ }^{\circ} \mathrm{C} / \mathrm{s}\right)$ under highvacuum conditions $\left(\sim 10^{-6}\right.$ Torr $)$ allowed the measurements to approach adiabatic conditions. The current and voltage through the sensors were measured and used subsequently for power, resistance, and temperature calculations. Calorimetric data are presented as a dependence of heat capacity on temperature. The first pulse resulted in a significantly different endotherm peak than subsequent pulses. For this reason, the calorimetry data presented in this article represent the first pulse separate from the subsequent pulses. For noise reduction, over 100 pulses after the first pulse were conducted and averaged during each experiment.

In the ideal case where the two sensors increase in temperature at exactly the same rate, the power required to melt the sample deposited on the sample calorimeter would simply be

$$
P(t)=V_{S} I_{S}-V_{R} I_{R}
$$

where $V$ and $I$ denote the voltages and currents, respectively, through the sample and reference (indices $\mathrm{S}$ and $\mathrm{R}$, respectively) sensors and $t$ is time. The heat capacity would then be

$$
C_{P}(T)=\frac{P[T(t)]}{d T / d t}
$$

However, our system deviates from the ideal case because of several conditions. An in-depth discussion of the details of the data calculation to account for these nonideal conditions may be found in Zhang et al. ${ }^{22}$

To conduct the annealing experiments, the power supply was modified to apply discrete, short, low-current pulses incrementally so that a controlled temperature ramp rate was possible. By careful adjustment of the temperature, abrupt stoppage in the ramp rate was possible, and the system could be held at the desired annealing temperature for long or short times. In this work, the time at the annealing temperature was $1 \mathrm{~s}$.

\section{Crystal Growth}

The source material used in the experiments was a high-density linear PE with a density of 0.96 $\mathrm{g} / \mathrm{cm}^{3}$ and a melt index of 3.4 ; it was in the form of a blown film produced by Visking Co. around 1960. To obtain single crystals, we prepared a 5 $\times 10^{-4}$ wt $\%$ solution by dissolving PE in about 70 $\mathrm{mL}$ of xylene at $125{ }^{\circ} \mathrm{C}$. The solution was quenched in an ice-water bath, and a self-seeding method followed. ${ }^{23}$ The self-seeding method involves slowly heating the solution to a seeding temperature, $99{ }^{\circ} \mathrm{C}$, to dissolve most of the crystals formed upon quenching and leaving only a small amount of seeds in solution. After the selfseeding, the solution was transferred to a beaker in an oil bath, and the crystals were grown isothermally at $70 \pm 1{ }^{\circ} \mathrm{C}$. Although crystal growth may have been initiated during the cooling to 70 ${ }^{\circ} \mathrm{C}$, there was no evidence for a thicker center in the crystals. The crystals were sprayed onto the calorimeter with an airbrush unit. Care was taken to spray at a low velocity and for short times to avoid damaging the crystals in flight and ensure the rapid evaporation of the solvent. After deposition of the sample, the calorimetric cells were pumped down to $10^{-6}$ Torr in an evaporation chamber with a diffusion pump, and calorimetric measurements were performed.

\section{AFM}

Because the sample size was so small, using a microbalance was not feasible in determining the mass. Thus, the mass was approximated from the volume as determined with a conventional optical microscope in conjunction with a Digital Instruments Nanoscope III atomic force microscope. From the optical microscope, we obtained the number of crystals present on the sample sensor. From AFM imaging, we obtained the volume of individual crystals by determining the shape, lateral dimensions, and thickness. The number of crystals multiplied by the volume of a typical crystal yielded the total volume of material on the sensor. We followed the same method as Nakagawa et al. ${ }^{24}$ to obtain images with the AFM. ${ }^{24}$ The samples were observed in contact mode with a V-shaped silicon nitride cantilever with an integrated pyramidal tip. The cantilever had a length of $200 \mu \mathrm{m}$ and a spring constant of 0.12 $\mathrm{N} / \mathrm{m}$. The images were obtained in constant force mode with an applied force of about $10 \mathrm{nN}$. After image correction for image tilting and bowing, the lamellar thickness was measured by section profile and depth analysis tools provided by Digital Instruments. 


\section{TEM}

For electron microscopy, the crystals were deposited on a silicon nitride membrane (similar to the TDSC sensor but without the heater portion). No additional coating or staining was done on the crystals. Diffraction and bright field images were obtained for several crystals before and after calorimetric measurements. As expected, ${ }^{25}$ the diffraction patterns lasted a very short time. The crystals were examined with a Phillips CM-12 transmission electron microscope at $120 \mathrm{keV}$.

\section{RESULTS AND DISCUSSION}

Figure 2 shows an AFM image of a typical PE single crystal studied in our experiments. The thin rim visible in the AFM micrograph most likely developed during the subsequent cooling of the suspension to room temperature. Our images of the single crystals were similar to those obtained by Kawaguchi et al. ${ }^{5}$ and Kajiyama et al. ${ }^{26}$ with similarly grown crystals. The majority of the crystals were lozenge-shaped with a length of 20 $\mu \mathrm{m}$, a width of $13 \mu \mathrm{m}$, and a thickness of $12 \pm 1$ $\mathrm{nm}$. Some of the lozenge crystals, however, had spiral growths. Other crystals were star-shaped and had multiple layers. No matter what the crystal shape, AFM measurements yielded consistent lamellar thicknesses. Several crystals of each shape were imaged and analyzed so that a volume could be assigned to each shape type. Hence, each shape type-lozenge-shaped, lozenge-shaped with spiral growths, and star-shaped-were imaged and counted. In this way, we determined the total volume of sample detected by the calorimeter.
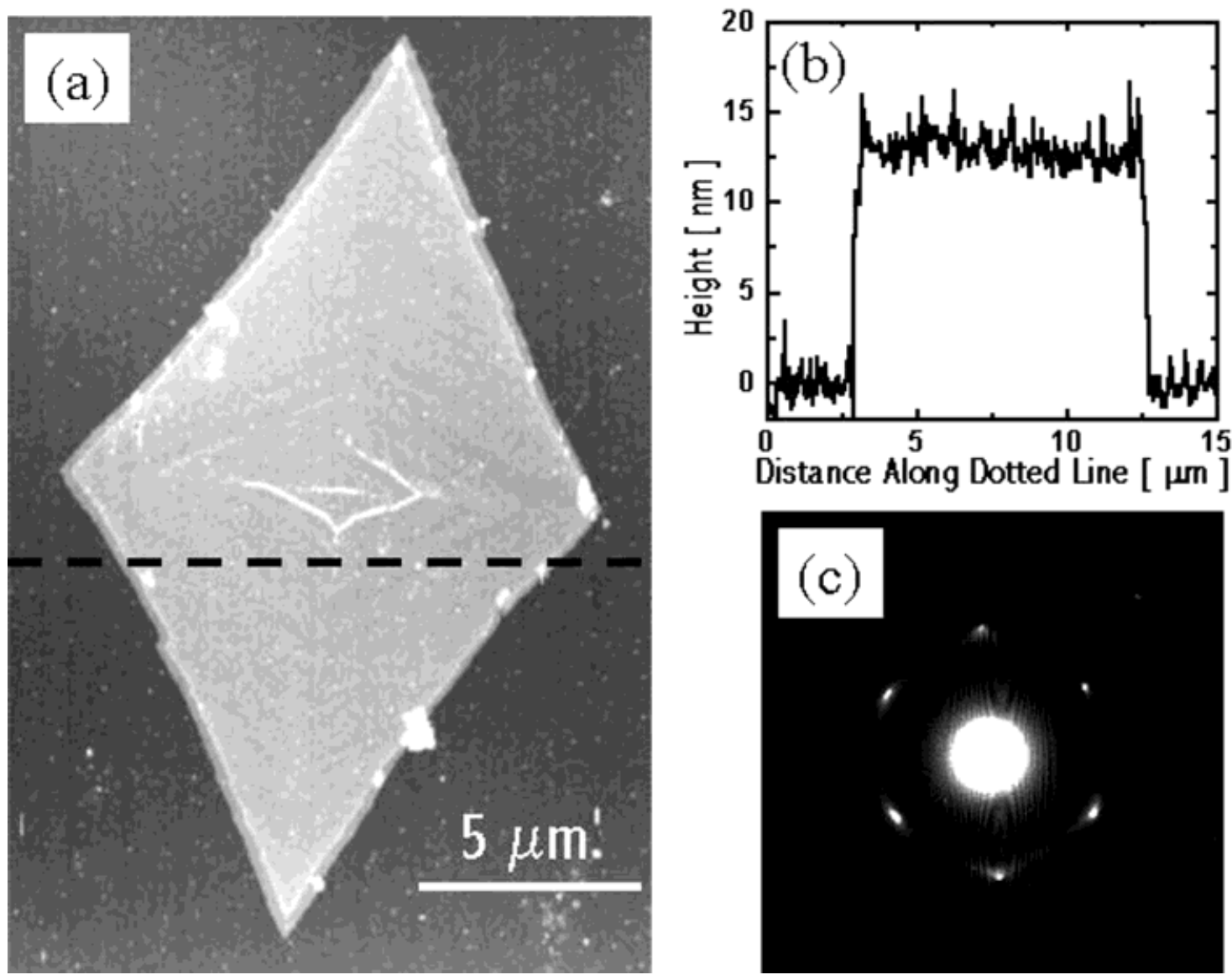

Figure 2. (a) AFM micrograph of a typical $20 \mu \mathrm{m} \times 13 \mu \mathrm{m} \times 12 \mathrm{~nm}$ single crystal. The pleats near the center of the crystal are a result of the original tent-shaped crystal collapsing on the flat silicon nitride surface. (b) The section profile of the crystal. The $x$ axis of the profile corresponds to the dotted line in part A. (c) A diffraction pattern of a similar crystal obtained by TEM. 
To arrive at the sample mass, we multiplied the total volume of crystals by the density of the material used for fabricating the original film $\left(0.96 \mathrm{~g} / \mathrm{cm}^{3}\right)$, assuming the density of the single crystals was equal. In addition, calculating the sample mass with the volume is also complicated by the error associated with volume calculation and counting. These errors can contribute an additional $15 \%$ uncertainty. If enough data are taken with various amounts of crystals, the sample mass calculated in this manner provides a means of verifying our calorimetric measurements because the thermal properties of heat capacity and heat of fusion are extensive: they scale with amount.

Figure 3(a) shows the normalized results for a 30-crystal experiment. Also shown is the specific heat capacity of the bulk material (obtained with conventional DSC) for comparison. The endotherm from the as-deposited scan indicates the $T_{\mathrm{m}}$ of our single-crystal $\mathrm{PE}$ is $123 \pm 2{ }^{\circ} \mathrm{C}$. This value is about $9{ }^{\circ} \mathrm{C}$ less than that of the bulk. Previous work has also determined the $T_{\mathrm{m}}$, albeit not by calorimetry, of $\mathrm{PE}$ single crystals grown from solution. Table I summarizes the results for comparison with our work. Our work agrees well with optical microscopy experiments on similarly grown crystals conducted by Hellmuth and Wunderlich. ${ }^{12}$ Their experiments consisted of several trials of monitoring changes in appearance during heating with an optical interference microscope. Their samples were growth spirals of single-crystal PE consisting of 100-200 lamellae with a total thickness of $2000 \mathrm{~nm}$, grown from a dilute solution of toluene. At heating rates of 2-33 ${ }^{\circ} \mathrm{C} / \mathrm{s}$, the $T_{\mathrm{m}}$ was $121 \pm 2{ }^{\circ} \mathrm{C}$. Our $T_{\mathrm{m}}$ also agrees with the results of Alamo and Mandelkern. ${ }^{20}$ Their experiments consisted of studying the crystallite thickness distribution as determined by Raman spectroscopy of a sample annealed at different temperatures $\left(112-150{ }^{\circ} \mathrm{C}\right)$. They concluded that the melting of powders of solutiongrown PE [weight-average molecular weight $\left(M_{\mathrm{w}}\right)$ $=166,000$, weight-average molecular weight/ number-average molecular weight $\left(M_{\mathrm{w}} / M_{\mathrm{n}}\right)$ $=1.11$ s single crystals, with a thickness of about $13 \mathrm{~nm}$, occurs below $125^{\circ} \mathrm{C}$.

Another important characteristic of the single crystals, apparent in Figure 3(a), is the difference in the endotherm peak of the first pulse and the subsequent pulses. There is a $50 \%$ reduction in heat of fusion from the as-deposited crystals to subsequent quenched crystals. Also, there is a shift in the peak of $7^{\circ}$. This may be attributed to the fast cooling, essentially quenching, of the PE crystals from a molten state to a solid state. The cooling occurs within several milliseconds. The phenomenon was also reported by Grubb et al. ${ }^{4}$ During their experiments, using real-time smallangle X-ray scattering studies with the annealing of PE single crystals, they noticed that heating occurred much more slowly on the first ramp than on the subsequent ramp. They attributed this to the greater amount of endothermic reorganization that occurred on the first heating. Therefore, it was suspected that after the initial melting, there was a loss of crystallinity upon cooling. This was confirmed with TEM. Figure 2(c) is a diffraction pattern obtained by TEM before any heating. After heating, no diffraction pattern was observed.

We obtained consistent values for the latent heat of fusion, $\Delta H_{\mathrm{f}}( \pm 15 \%)$ over a wide range of heating rates. Figure $3(\mathrm{~b})$ shows the endotherm for the 30-crystal experiment at heating rates of $20,000-100,000{ }^{\circ} \mathrm{C} / \mathrm{s}$. In the subsequent pulses, the peak of the endotherm remained constant at about $116{ }^{\circ} \mathrm{C}$ as the rate increased. The full width at half-maximum of the peak increased from 6 to $10^{\circ}$. This may be due to the limitations of the instrument; at higher heating rates, the temperature resolution is not as good as at low heating rates, and so during calculations to arrive at an endotherm a broader peak may arise. We do not believe either superheating or thermal lag is occurring because although the peak may widen, it does not shift to higher temperatures.

We calculated the latent heat of melting by integrating the area under the heat capacity peak of the first scan. Figure 4 shows the heat of fusion of 25-2000 single crystals as a function of mass calculated from volume for the given amount of crystals. Also depicted is the heat of fusion (solid and dashed lines) with the same specific latent heat of melting of the bulk assumed (as measured by conventional DSC). Taking the slope of the raw data in Figure 4 yields a specific latent heat of melting for PE single crystals of $198 \pm 10 \mathrm{~J} / \mathrm{gm}$, which is $40 \%$ greater than the bulk value, 140 J/gm.

The measured heat capacity (between 40 and $60{ }^{\circ} \mathrm{C}$ ) also scales in relation to the sample mass. The specific heat capacity calculated in the same manner as the specific latent heat of melting (taking the slope of the measured heat capacity of the isolated single crystals with respect to mass) yields a specific heat capacity of $2.1 \pm 0.3 \mathrm{~J} / \mathrm{gm} \mathrm{K}$. This value is within $25 \%$ of the bulk value, 1.7 

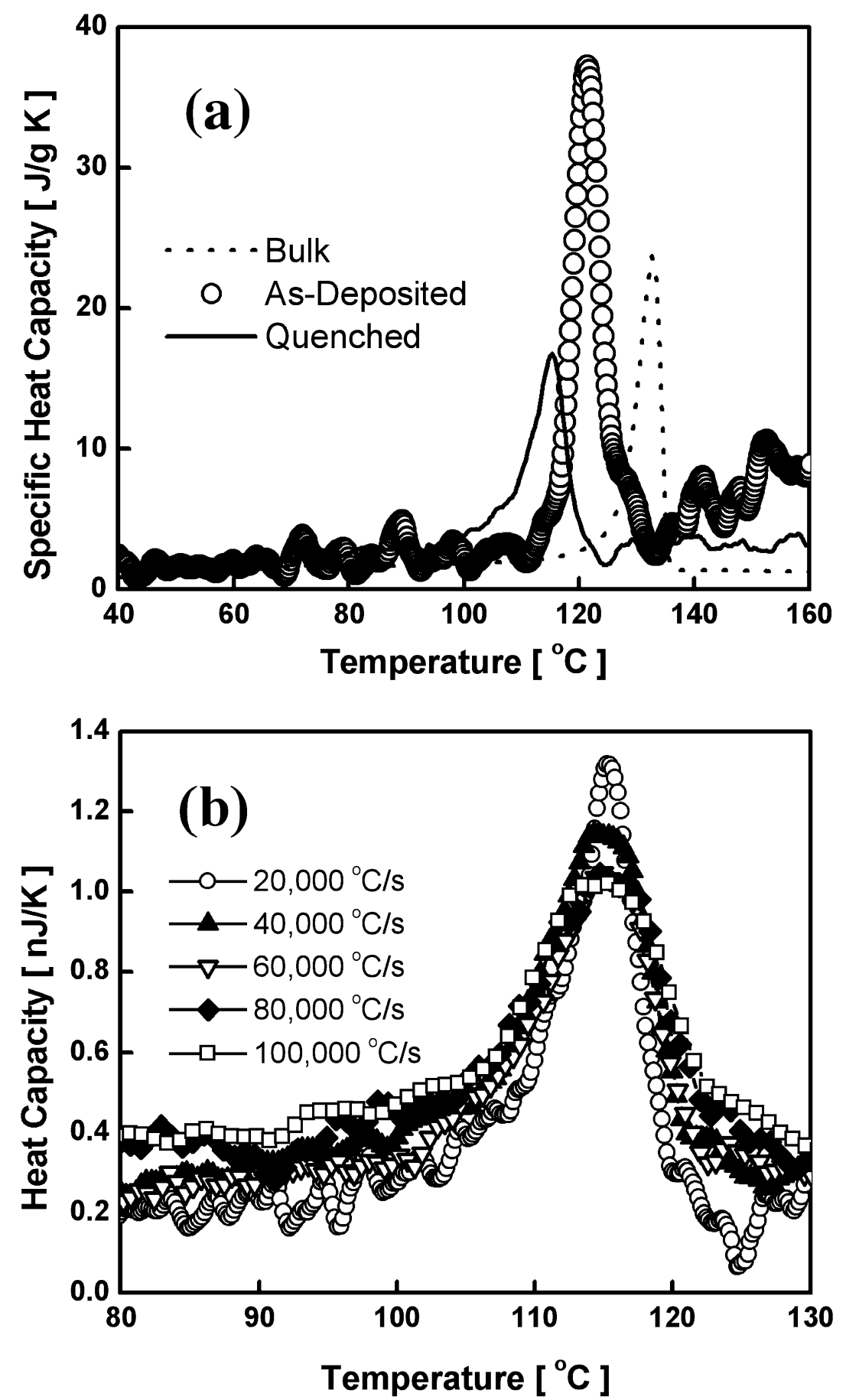

Figure 3. (a) The specific heat capacity for the source material from the first pulse and the subsequent pulses for 30 crystals at a heating rate of $20,000{ }^{\circ} \mathrm{C} / \mathrm{s}$ is shown. For clarity, the as-deposited and quenched scans were smoothed with a 15-point adjacent averaging. (b) Also shown is the endotherm of the quenched scans at various heating rates. Its position and shape does not change significantly with the heating rate. For clarity, the data have undergone a 5-point adjacent average smoothening.

$\mathrm{J} / \mathrm{gm} \mathrm{K}$ (as determined by DSC). With lower numbers of crystals, the effects of handling and possible contamination during crystal deposition are more pronounced. These effects contribute to uncertainties and broad variations in the overall baseline of the system (specific heat capacity); 
Table I. Comparisons with Other Works

\begin{tabular}{|c|c|c|c|c|c|}
\hline & Source Material & Lamellar Thickness & Method of Analysis & $T_{m}$ & Reference \\
\hline This work & $\begin{array}{l}\text { Density of } 0.96 \mathrm{~g} / \mathrm{cm}^{3} \\
\text { melt index of } 3.4\end{array}$ & $12 \pm 1 \mathrm{~nm}$ & DSC & $123 \pm 2{ }^{\circ} \mathrm{C}$ & \\
\hline $\begin{array}{l}\text { Hellmuth and } \\
\text { Wunderlich }\end{array}$ & $M_{w}=80,000$ & $10-20 \mathrm{~nm}$ & $\begin{array}{l}\text { Optical } \\
\text { interference } \\
\text { microscopy }\end{array}$ & $121 \pm 2{ }^{\circ} \mathrm{C}$ & 12 \\
\hline $\begin{array}{l}\text { Alamo and } \\
\text { Mandelkern }\end{array}$ & $M_{w}=166,000$ & $\sim 13 \mathrm{~nm}$ & $\begin{array}{l}\text { Annealing followed } \\
\text { by Raman } \\
\text { spectroscopy }\end{array}$ & $<125{ }^{\circ} \mathrm{C}$ & 20 \\
\hline
\end{tabular}

however, they do not affect the calculation of the heat of fusion.

Thus far, we have been able to observe the effects of annealing of the quenched crystals obtained after the first pulse. Figure 5 shows that the 1-s annealing did not yield an endotherm with the same area under the peak or peak position as the first pulse endotherm when the quenched crystals were annealed at different temperatures. However, we did observe a significant change in the heat of fusion (an increase of over $50 \%$ ) and peak position (an increase of up to $15{ }^{\circ} \mathrm{C}$ ) from that of the unannealed quenched crystals by annealing at different temperatures. Furthermore, the systems always reverted back to the endotherm of the quenched state after any pulsing. In future work, we plan to conduct annealing on the as-deposited crystals.

The lowest amount of PE detected was 25 crystals. This is pushing the limits of TDSC in its present form. The surface coverage for 25 crystals on this sensor area is less than $0.1 \%$. By decreas-

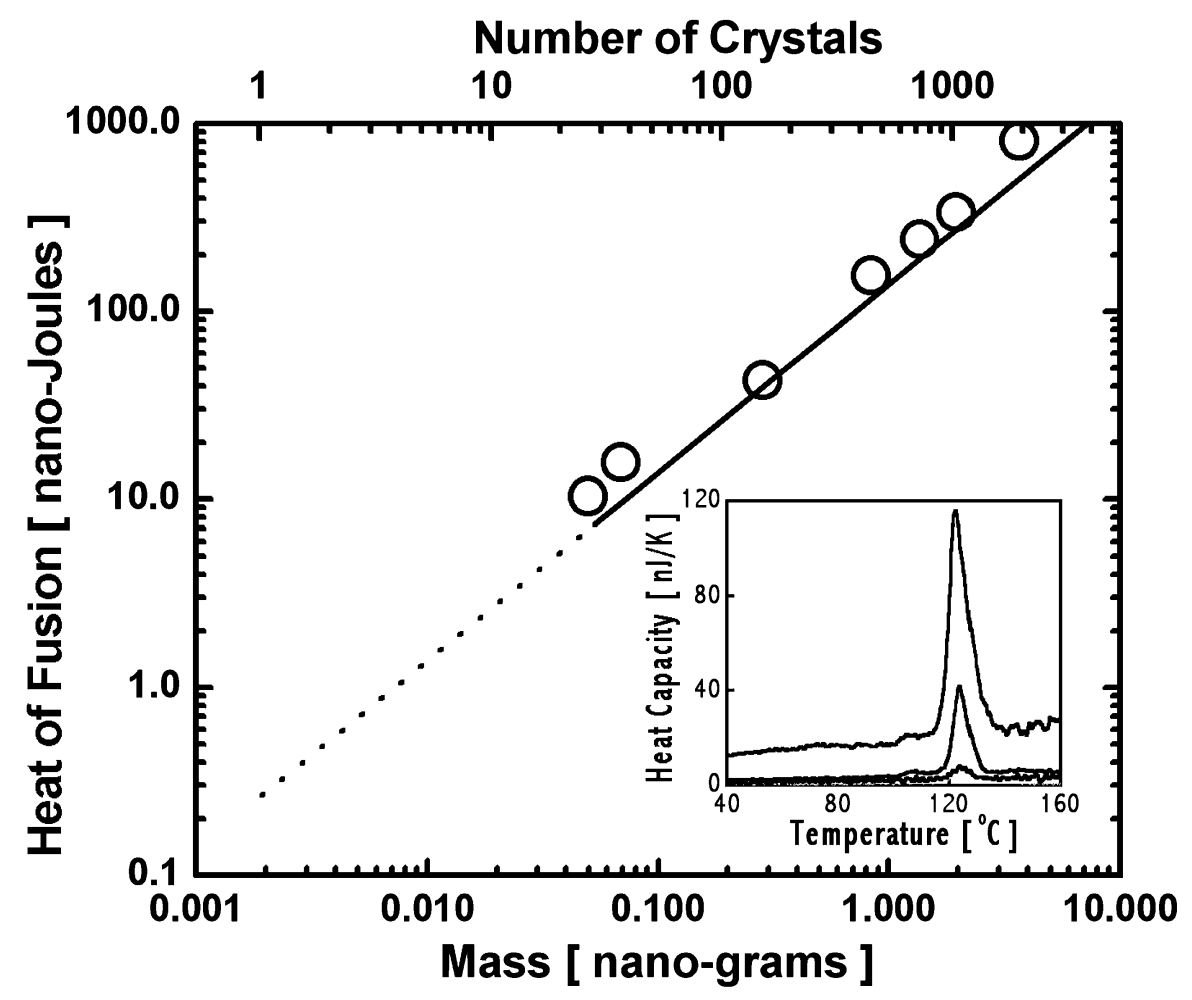

Figure 4. Heat of fusion of 25-2000 PE single crystals determined by nanocalorimetry $(\bigcirc)$. The bottom axis indicates the mass as determined by volume calculations. The top axis indicates the approximate number of PE single crystals. The solid and dashed lines indicates the expected heat of fusion with the bulk value. The inset graph shows the heat capacity of 120,680 , and 2000 PE single crystals. 

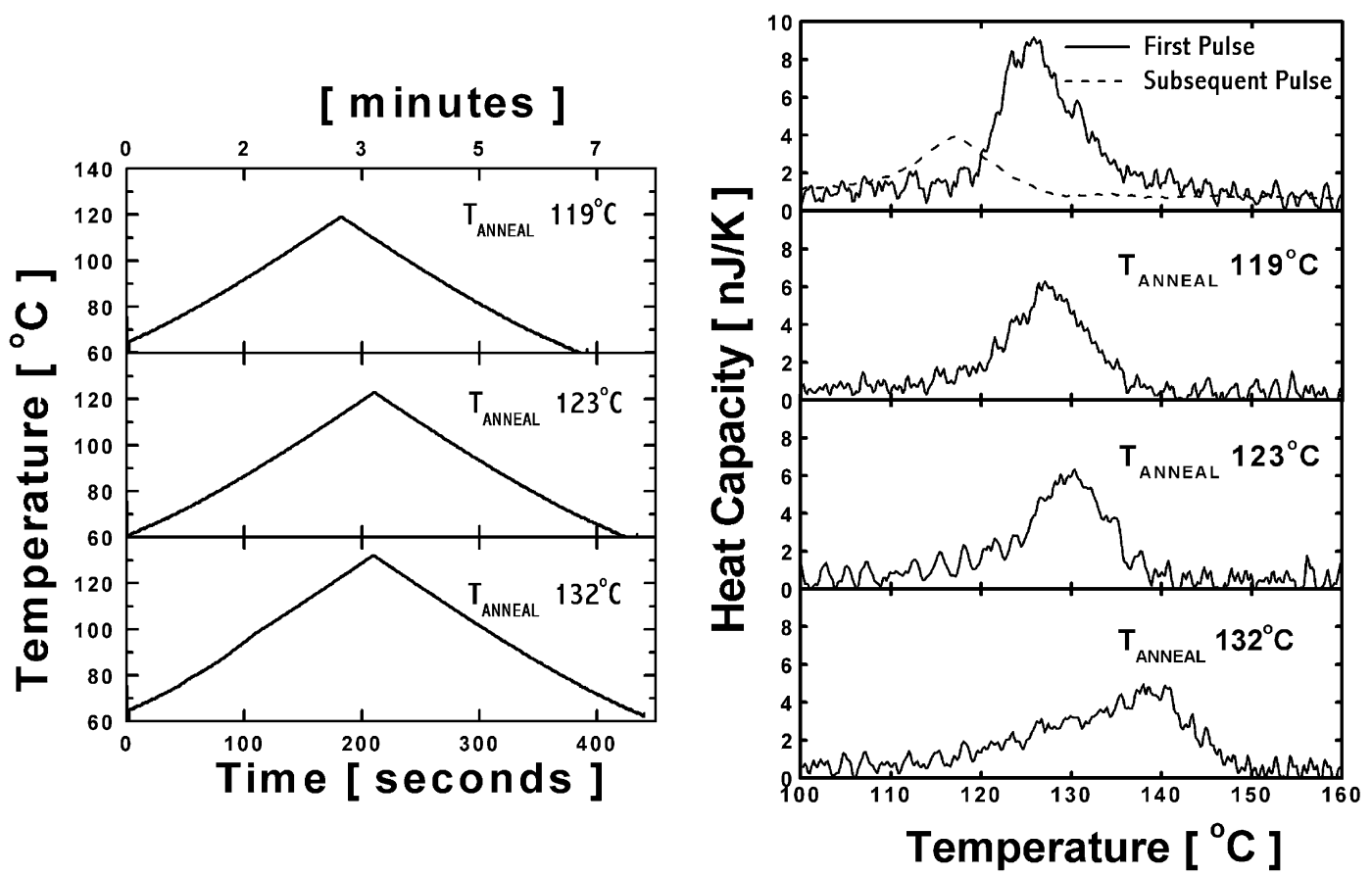

Figure 5. (a) Annealing scheme employed for each caloric curve. The same set of crystals was used for each trial. (b) The endotherm shifts to a higher temperature on annealing. However, the heat of fusion never reaches that of the first pulse. All annealing was conducted on quenched crystals. After any calorimetry, the caloric curve returned to that of quenched crystals, indicated by the dashed line.

ing the sensor area, we should increase the sensitivity to allow the detection of the melting of a single crystal. This has strong implications for the world of polymer science. It may then be possible to probe the properties of individual crystals as opposed to merely the average of an aggregate of crystals.

\section{CONCLUSION}

In summary, we used a nanocalorimeter to study the melting of PE single crystals. The $T_{\mathrm{m}}$, which previously had not been determined by calorimetry, and the heat of fusion were measured with good confidence. With this technique, the detection of the melting of as few as 25 crystals is possible. Future work with the probing of 1 single crystal may be possible with a redesign of the sensor. Future work will also include investigating the effect of annealing on the thermodynamics of the PE single-crystal system.

The authors gratefully acknowledge all the staff, especially Phil Infante, at the Cornell Nanofabrication Fa- cility for their help in fabricating the calorimeters. Microanalysis was performed at the Center for Microanalysis of Materials at the University of Illinois at Urbana-Champaign. A. T. Kwan and M. Y. Efremov were partially funded by the American Chemical Society-Petroleum Research Fund (\#33580-AC7). L. H. Allen was supported by the National Science Foundation (NSF-DMR 0108694). Equipment support was contained in NSF-DMR 9803019. F. Scheittekatte is grateful to the Natural Sciences and Engineering Research Council of Canada for its financial support.

\section{REFERENCES AND NOTES}

1. Lai, S. L.; Ramanath, G.; Allen, L. H. Appl Phys Lett 1997, 70, 43.

2. Zhou, H.; Wilkes, G. L. Polymer 1997, 38, 5735.

3. Snyder, R. G.; Scherer, J. R.; Reneker, D. H.; Colson, J. P. Polymer 1982, 23, 1286.

4. Grubb, D. T.; Liu, J. J.-H.; Caffrey, M.; Bilderback, D. H. J Polym Sci Polym Phys Ed 1984, 22, 367.

5. Kawaguchi, A.; Ichida, T.; Murakami, S.; Katayama, K. Colloid Polym Sci 1984, 262, 597.

6. Mandelkern, L.; Sharma, R. K.; Jackson, J. F. Macromolecules 1969, 2, 644 . 
7. Magill, J. H. J Polym Sci Polym Lett Ed 1982, 20, 1.

8. Van Oort, M. J. M.; White, M. A. Rev Sci Instrum 1987, 58, 1239.

9. Pecharsky, V. K.; Moorman, J. O.; Gschneidner, K. A., Jr. Rev Sci Instrum 1997, 68, 4196.

10. Leung, W. M.; Manley, R. St. J.; Panaras, A. R. Macromolecules 1985, 18, 746.

11. Lai, S. L.; Guo, J. Y.; Petrova, V.; Ramanath, G.; Allen, L. H. Phys Rev Lett 1996, 77, 99.

12. Hellmuth, E.; Wunderlich, B. J Appl Phys 1965, 36, 3039 .

13. Efremov, M. Y.; Schiettekatte, F.; Zhang, M.; Olson, E. A.; Kwan, A. T.; Berry, R. S.; Allen, L. H. Phys Rev Lett 2000, 85, 3560.

14. Denlinger, D. W.; Abarra, E. N.; Allen, K.; Rooney, P. W.; Messer, M. T.; Watson, S. K.; Hellman, F. Rev Sci Instrum 1994, 65, 946.

15. Nakagawa, Y.; Schäfer, R.; Güntherodt, H.-J. Appl Phys Lett 1998, 73, 2296.

16. Olson, E. A.; Efremov, M. Y.; Kwan, A. T.; Lai, S.; Petrova, V.; Schiettekatte, F.; Warren, J. T.; Zhang, M.; Allen, L. H. Appl Phys Lett 2000, 77, 2671.
17. Fominaya, F.; Fournier, T.; Gandit, P.; Chaussy, J. Rev Sci Instrum 1997, 68, 4191.

18. Riou, O.; Gandit, P.; Charalambous, M.; Chaussy, J. Rev Sci Instrum 1997, 68, 1501.

19. Weaver, T. J.; Harrison, I. R. Polymer 1981, 22 , 1590.

20. Alamo, R.; Mandelkern, L. J Polym Sci Part B: Polym Phys 1986, 24, 2087.

21. Sadler, D. M.; Spells, S. J. Macromolecules 1989, $22,3941$.

22. Zhang, M.; Efremov, M. Y.; Schiettekatte, F.; Olson, E. A.; Kwan, A. T.; Lai, S. L.; Wisleder, T.; Greene, J.; Allen, L. H. Phys Rev B 2000, 62, 10548.

23. Blundell, D. J.; Keller, A. J Polym Sci B 1968, 6, 433.

24. Nakagawa, Y.; Hayashi, H.; Takahagi, H.; Soeda, F.; Ishitani, A.; Toda, A.; Miyaji, H. Jpn J Appl Phys 1994, 33, 3771.

25. Geil, P. H. Polymer Single Crystals; Wiley: New York, 1963; p 24.

26. Kajiyama, T.; Tanaka, K.; Ge, S-R.; Takahara, A. Prog Surf Sci 1996, 52, 1. 